\title{
Acute psychiatric inpatient care: A cross-cultural comparison between two hospitals in Germany and Japan
}

International Journal of

Social Psychiatry

59(8) $77 \mid-78$ I

(c) The Author(s) 2012

Reprints and permissions:

sagepub.co.uk/journalsPermissions.nav

DOI: I0.II77/00207640I2456808

isp.sagepub.com

(SAGE

\author{
Kumi Moriwaki,' Tanja Neuner, ${ }^{2}$ Bettina Hübner-Liebermann, ${ }^{2}$ \\ Helmut Hausner, ${ }^{2}$ Markus Wittmann, ${ }^{2}$ Toshihiro Horiuchi, ${ }^{3}$ \\ Hiromi Watanabe, ${ }^{3}$ Hideyuki Kato, ${ }^{3}$ Junich Hirakawa ${ }^{3}$ and \\ Kazumasa Iwai ${ }^{4}$
}

\begin{abstract}
Background: Intercultural differences influence acute inpatient psychiatric care systems.

Aims: To evaluate characteristics of acute inpatient care in a German and a Japanese hospital.

Method: Based on a sample of 465 admissions to the Psychiatric State Hospital Regensburg (BKR) and 91 admissions to the Hirakawa Hospital $(\mathrm{HH})$ over a six-month period in 2008 , data from the psychiatric basic documentation system (BADO) were analysed with regard to socio-demographic characteristics, treatment processes and outcome indicators. Results: Schizophrenia and related psychosis was the most common diagnosis in both hospitals. Cases at the BKR were admitted more quickly after onset of the present episode. Global Assessment of Psychosocial Functioning (GAF) ratings at admission were lower at the $\mathrm{HH}$. Most admissions to both hospitals received psychopharmacological treatment, but more at the $\mathrm{HH}$ received psychotherapy. Length of stay was significantly longer at the $\mathrm{HH}$ ( 75 days) than at the BKR (28 days). Admissions to the $\mathrm{HH}$ were more improved with regard to $\mathrm{GAF}$ and clinical global impression (CGI).

Conclusions: Acute admissions in Germany provide intensive care with short hospitalization as crisis intervention. For acute admissions in Japan, comprehensive care for severe mental illness precedes emergency admissions and achieves greater improvement with longer hospitalization.
\end{abstract}

\section{Keywords}

Psychiatric emergency services, acute psychiatric inpatient care, cross-cultural comparison, health care quality assurance, routine data

\section{Introduction}

Since the 1950s, deinstitutionalization of mental health services has been widespread in western countries, and such services have become better established in the community. Although this shift from hospital-based to community-based services has been accompanied by a reduction in hospital beds and a shortened duration of inpatient care, there have also been negative consequences (MunkJørgensen, 1999). The critical demand for acute psychiatric inpatient services is reflected by the increase in compulsory admissions (Priebe et al., 2008; Salize \& Dressing, 2004) and readmissions (Appleby et al., 1993; Goldstein \& Shemansky, 2000). In contrast, in Japan, the number of psychiatric beds increased between the 1960s and 1990s and was associated with longer length of stay relative to other countries (Shinfuku, 1998). The lack of community support systems and the stigma attached to psychiatric patients promoted hospital-based care (Shinfuku, 1998).
However, in the mid-1980s, deinstitutionalization and community-based services began to gain attention in Japan as well (Oshima, Mino \& Inomata, 2003). Many psychiatric hospitals have changed their focus from chronic to acute inpatient care. Japanese psychiatric services are now in the

'Department of Psychiatry, Tokyo Women's Medical University, Medical Centre East, Tokyo, Japan

${ }^{2}$ Department of Psychiatry, Psychosomatics and Psychotherapy,

University of Regensburg, Germany

${ }^{3}$ Hirakawa Hospital, Tokyo, Japan

${ }^{4}$ Kanagawa Prefectural Hospital Organization, Kanagawa Psychiatric

Centre, Kinko Hospital, Kanagawa, Japan

\section{Corresponding author:}

Kumi Moriwaki, Department of Psychiatry, Tokyo Women's Medical University, Medical Centre East, Japan, 2-I-I0 Nishiogu, Arakawa-ku, Tokyo I I6-8567, Japan.

Email: kumi.moriwaki@googlemail.com 
middle of a transition from hospital based to community based (Ito, 2009).

Along with this reform process, quality assurance in mental health care has gained importance. The psychiatric basic documentation system (BADO) recommended by the German Society of Psychiatry and Psychotherapy (DGPPN) (Cording, 1998) has contributed to quality assurance in psychiatric inpatient care (Spießl, Hübner-Liebermann, Cording \& Klein, 2004). Awareness of the importance of promoting the reform process in Japan has led to the translated version of the DGPPN-BADO (J-BADO) being introduced in some Japanese hospitals (Moriwaki, Iwai \& Cording, 2011). Based on BADO data in 1997, our group conducted the first international and intercultural comparison between Germany and Japan with regard to treatment of schizophrenia (Hübner-Liebermann, Spießl, Iwai \& Cording, 2005). The results revealed differences in service provision and suggested the need for the introduction of multidimensional treatment and the extension of community-based services in Japan.

The aim of this study is to evaluate aspects of acute psychiatric inpatient services in a German and a Japanese hospital by using BADO data to examine potential differences in service provision and to define the role of acute inpatient services in the two countries.

\section{Methods}

\section{Subjects}

The hospital comparison is based on data of the DGPPNBADO for all admissions to the acute psychiatric wards of the Psychiatric State Hospital Regensburg (BKR), Germany, and to the Hirakawa Hospital (HH), Japan, from 1 July to 31 December 2008. The BKR is a universityaffiliated psychiatric hospital with about 5,000 admissions per year, caring for a catchment area of about 800,000 inhabitants ( 0.6 beds per 1,000 population). The 475 beds are located in 21 wards, including two acute psychiatric wards (closed, mixed-sex) with a total of 53 beds. Acute psychiatric wards are obligated to take acute cases around the clock. The HH is a private psychiatric hospital located in a suburb of Tokyo with about 400 admissions a year. It has seven wards with a total of 313 beds, including one acute psychiatric ward (closed, mixed-sex) with 44 beds. In Japan, about $80 \%$ of all psychiatric beds are provided in private hospitals (Shinfuku, 1998), although payment for patients at these hospitals is covered by public expenditure or national insurance schemes. In clinical practice, there is no distinction between public and private hospitals. For Tokyo with its 12 million inhabitants, there are about 24,000 psychiatric beds located in 114 psychiatric departments and hospitals ( 1.9 beds per 1,000 population). In the regional psychiatric emergency system, acute cases during the day are admitted in rotation by some of the private psychiatric hospitals, including the $\mathrm{HH}$ and at night by four public hospitals.

This study includes 394 patients with a total of 465 acute admissions to the BKR and 90 patients with a total of 91 acute admissions to the $\mathrm{HH}$.

\section{Instrument}

The DGPPN-BADO is a standardized assessment instrument. It consists of more than 70 socio-demographic and disease-related variables and variables regarding therapeutic process and outcome. These parameters are entered by the treating psychiatrist or psychologist for every patient admitted to the psychiatric hospital. The following variables were analysed:

- Socio-demographic variables: age, gender, marital status, housing situation, occupational status, initiator of admission, previous psychiatric hospitalization and legal basis of admission.

- Disease-related variables: psychiatric diagnoses, period since onset of the present episode, pre-treatment of the present episode, suicide attempt or selfharm before admission, aggressive behaviour before admission, Clinical Global Impression (CGI) and Global Assessment of Psychosocial Functioning (GAF) at admission.

- Therapeutic process: psychopharmacological treatment, psychotherapeutic treatment and psychosocial therapeutic measures.

- Outcome variables: type of discharge, CGI at discharge, GAF at discharge, length of stay and treatment after discharge.

- Critical events: problems related to psychopharmacological treatment, problems related to psychotherapeutic treatment, suicide attempt during stay, aggressive behaviour during stay and use of restraints (i.e. straps around the waist or the wrists and ankles).

\section{Statistical analysis}

We used univariate tests ( $t$-tests, $\chi^{2}$ tests) to compare the $\mathrm{BKR}$ to the $\mathrm{HH}$ with regard to the BADO variables. Analyses were performed using SPSS version 16.0. Using a Bonferroni correction, $\alpha$ was set at 0.001 .

\section{Results}

\section{Sociodemographic and clinical characteristics}

Admissions to the HH ( $M=47.7$ years) were significantly older than admissions to the BKR ( $M=39.8$ years) (Table 1$)$. Regarding gender, marital status and occupational status, no significant differences were found between the two 
hospitals. Admissions to the BKR were more likely to live in care homes or supported housing than admissions to the HH. Outpatient treatment for the present episode prior to admission was provided significantly less often in the BKR. Of those admitted, $43.1 \%$ at the BKR and $60.4 \%$ at the $\mathrm{HH}$ had received outpatient pre-treatment from a psychiatrist. Admissions to the BKR showed a significantly shorter period since onset of the present episode, with twothirds admitted within four weeks after onset of illness. Whereas admission by a doctor was most frequent at both hospitals, admission by the police, justice system or health authorities occurred significantly more often at the BKR. In contrast to the BKR, admission by psychosocial services was found significantly more often at the HH.

For $53.8 \%$ of admissions to the $\mathrm{HH}$, it was their first psychiatric inpatient stay. A total of $67.8 \%$ of admissions to the BKR had already had an inpatient stay, with the majority of those in the same hospital. Altogether, 49 patients in the BKR $(12.4 \%)$ were re-hospitalized during the index period: 35 patients twice, nine patients three times and five patients from four to eight times; this is in contrast to the $\mathrm{HH}$, which had only one re-hospitalized patient (1.1\%). The legal basis for stay differed significantly between the two hospitals, with $72.7 \%$ of admissions to the BKR being voluntary, compared to $52.7 \%$ of those at the $\mathrm{HH}$.

\section{Diagnostic group and severity of illness}

Schizophrenia and related psychosis represented the most common diagnostic group at admission in both hospitals, with mood disorders being the second most common (Table 2). The proportion of mood disorders was higher at

Table I. Socio-demographic and clinical characteristics of acute admissions.

\begin{tabular}{|c|c|c|c|c|c|c|}
\hline & \multirow{2}{*}{\multicolumn{2}{|c|}{$\begin{array}{l}\mathrm{BKR}(N=465) \\
M \pm S D\end{array}$}} & \multirow{2}{*}{\multicolumn{2}{|c|}{$\begin{array}{l}\mathrm{HH}(\mathrm{N}=9 \mathrm{l}) \\
M \pm \mathrm{SD}\end{array}$}} & \multicolumn{2}{|l|}{ t-test } \\
\hline & & & & & $t$ & $p$ \\
\hline \multirow[t]{3}{*}{ Age (years) } & \multicolumn{2}{|c|}{$39.8 \pm 13.3$} & \multicolumn{2}{|c|}{$47.7 \pm 19.7$} & -3.70 & $.000 * * *$ \\
\hline & \multicolumn{2}{|c|}{$\operatorname{BKR}(N=465)$} & \multicolumn{2}{|c|}{$\mathrm{HH}(\mathrm{N}=91)$} & \multicolumn{2}{|l|}{$\chi^{2}$ test } \\
\hline & $n$ & $\%$ & $n$ & $\%$ & $\chi^{2}$ & $p$ \\
\hline \multicolumn{7}{|l|}{ Gender } \\
\hline Male & 245 & 52.7 & 33 & 36.3 & \multirow[t]{2}{*}{8.21} & \multirow[t]{2}{*}{.004} \\
\hline Female & 220 & 47.3 & 58 & 63.7 & & \\
\hline \multicolumn{7}{|l|}{ Marital status } \\
\hline Single & 253 & 60.5 & 38 & 41.8 & \multirow[t]{3}{*}{12.13} & \multirow[t]{3}{*}{.004} \\
\hline Married, cohabitating & 86 & 20.6 & 32 & 35.2 & & \\
\hline Separated, divorced, widowed & 79 & 18.9 & 21 & 23.1 & & \\
\hline \multicolumn{7}{|l|}{ Housing situation } \\
\hline At home & 304 & 81.7 & 84 & 92.3 & \multirow[t]{5}{*}{29.98} & \multirow[t]{5}{*}{$.000 * * *$} \\
\hline Care home or supported housing & 56 & 15.1 & I & I.I & & \\
\hline Psychiatric hospital & 0 & 0 & 3 & 3.3 & & \\
\hline Homeless & 11 & 3.0 & I & I.I & & \\
\hline Others & 1 & 0.3 & I & 2.2 & & \\
\hline \multicolumn{7}{|l|}{ Occupational status } \\
\hline Currently unemployed & 75 & 24.4 & 28 & 32.9 & \multirow[t]{5}{*}{8.52} & \multirow{5}{*}{.074} \\
\hline Full- or part-time employment & 74 & 24.1 & 17 & 20.0 & & \\
\hline Occasional or supported employment & 23 & 7.5 & 2 & 2.4 & & \\
\hline Pension (social, handicap, senior) & 83 & 27.0 & 17 & 20.0 & & \\
\hline Others (i.e. housewife, student) & 52 & 16.9 & 21 & 24.7 & & \\
\hline \multicolumn{7}{|l|}{ Pre-treatment of the present episode } \\
\hline \multicolumn{7}{|l|}{ Outpatient care } \\
\hline None & 137 & 39.9 & 7 & 7.7 & 33.74 & $.000 * * *$ \\
\hline By a psychiatrist & 148 & 43.1 & 55 & 60.4 & 8.64 & .003 \\
\hline By a psychotherapist & 10 & 2.9 & 0 & 0 & 2.72 & .099 \\
\hline By a GP & 23 & 6.7 & II & 12.1 & 2.89 & .089 \\
\hline At advice centre & 2 & 0.6 & 0 & 0 & 0.53 & .465 \\
\hline \multicolumn{7}{|l|}{ Inpatient care } \\
\hline At the present psychiatric hospital & 9 & 2.6 & 4 & 4.4 & 0.78 & .254 \\
\hline At another psychiatric hospital & 10 & 2.9 & 10 & 11.0 & 10.64 & .001 \\
\hline At a general hospital & 19 & 5.5 & 8 & 8.8 & 1.30 & .465 \\
\hline
\end{tabular}


Table I. (Continued)

\begin{tabular}{|c|c|c|c|c|c|c|}
\hline & \multicolumn{2}{|c|}{$\operatorname{BKR}(N=465)$} & \multicolumn{2}{|c|}{$\mathrm{HH}(\mathrm{N}=91)$} & \multicolumn{2}{|l|}{$\chi^{2}$ test } \\
\hline & $n$ & $\%$ & $n$ & $\%$ & $\chi^{2}$ & $p$ \\
\hline \multicolumn{7}{|l|}{ Period since onset of the present episode } \\
\hline Less than one week & 108 & 33.1 & 10 & 11.0 & 60.94 & $.000 * * *$ \\
\hline One week up to four weeks & 107 & 33.0 & 16 & 17.6 & & \\
\hline Four weeks up to three months & 37 & 11.4 & 39 & 42.9 & & \\
\hline Three months up to six months & 16 & 4.9 & 8 & 8.8 & & \\
\hline Six months up to one year & 12 & 3.7 & 7 & 7.7 & & \\
\hline More than one year & 44 & 13.6 & 44 & 12.1 & & \\
\hline \multicolumn{7}{|l|}{ Initiator of admission } \\
\hline Doctor & 180 & 47.9 & 54 & 59.3 & 3.86 & .050 \\
\hline Psychosocial service & 0 & 0 & 10 & 11.0 & 42.22 & $.000 * * *$ \\
\hline Police, justice system, health authorities & 112 & 29.8 & 9 & 9.9 & 15.11 & $.000 * * *$ \\
\hline Relatives & 36 & 9.6 & 8 & 8.8 & 0.05 & .818 \\
\hline Patient & 68 & 17.6 & 4 & 4.4 & 9.95 & .002 \\
\hline \multicolumn{7}{|l|}{ Previous psychiatric hospitalization } \\
\hline None & III & 32.2 & 49 & 53.8 & 50.17 & $.000 * * *$ \\
\hline Present hospital & 199 & 57.7 & 16 & 17.6 & & \\
\hline Other hospital & 35 & 10.1 & 25 & 28.6 & & \\
\hline \multicolumn{7}{|l|}{ Legal basis of stay } \\
\hline Voluntary & 309 & 72.7 & 48 & 52.7 & 19.67 & $.000 * * *$ \\
\hline Compulsory & 96 & 22.6 & 41 & 45.1 & & \\
\hline Other & 20 & 4.7 & 2 & 2.2 & & \\
\hline
\end{tabular}

$* * * p<.001$.

the $\mathrm{HH}$, whereas substance use disorders, personality disorders and anxiety disorders were more frequent at the BKR. These results were consistent with diagnoses at discharge. GAF at admission was significantly lower at the $\mathrm{HH}$, while there was no significant difference in severity of illness (CGI) between the two hospitals. With regard to suicide attempt and/or self-harm and overt aggression before admission, there were no significant differences between the two hospitals.

\section{Treatment during stay}

A total of $80.5 \%$ of the admissions to the BKR and all admissions to the $\mathrm{HH}$ received psychopharmacological treatment (Table 3). Atypical antipsychotics and lowpotency typical antipsychotics were prescribed significantly more often at the $\mathrm{HH}$, but high-potency typical antipsychotics were significantly more often prescribed at the BKR. Selective serotonin re-uptake inhibitors (SSRIs) were the antidepressants most frequently prescribed in both hospitals, although significantly more often in the $\mathrm{HH}$. Anxiolytics and/or hypnotics, valproic acid and anticholinergic drugs were administered significantly more often at the HH. In contrast to the BKR (23.6\%), almost all cases in the $\mathrm{HH}(92.0 \%)$ received supportive psychotherapy. Cognitive behavioural therapy (CBT), psychoeducation or other psychotherapy were rarely performed at either hospital. Sports therapy was applied significantly more frequently at the $\mathrm{HH}$.

\section{Outcomes}

The average length of stay was significantly longer at the HH (28.4 vs 75.2 days) (Table 4). Significantly more admissions to the BKR were discharged in the first month after admission. With regard to suicide attempt and/or self-harm, overt aggression and restraint, there were no significant differences between the two hospitals. GAF at discharge was almost equal at both hospitals, but severity of illness (CGI Part I) at discharge was significantly higher for the BKR. Improvements in GAF ( $\triangle \mathrm{GAF})$ and CGI (Part II) were significantly greater at the $\mathrm{HH}$. In both hospitals, most inpatients were regularly discharged, and discharge against medical advice occurred rarely.

At both hospitals, about $90-95 \%$ of the cases did not need inpatient aftercare. Outpatient aftercare was initiated for most cases at both hospitals, although type of aftercare differed significantly by hospital. Admissions to the BKR were more often scheduled to be treated by a psychiatrist in private practice or a general practitioner. In contrast, admissions to the $\mathrm{HH}$ were more often discharged to outpatient departments at their own hospital or other psychiatric hospitals. 
Table 2. Diagnosis and severity of illness at admission.

\begin{tabular}{|c|c|c|c|c|c|c|}
\hline & \multicolumn{2}{|c|}{$\operatorname{BKR}(N=465)$} & \multicolumn{2}{|c|}{$\mathrm{HH}(\mathrm{N}=91)$} & \multicolumn{2}{|l|}{$\chi^{2}$ test } \\
\hline & $n$ & $\%$ & $n$ & $\%$ & $\chi^{2}$ & $p$ \\
\hline \multicolumn{7}{|l|}{ Diagnosis at admission } \\
\hline $\begin{array}{l}\text { Organic brain disorders (including } \\
\text { dementia) }\end{array}$ & 13 & 3.4 & 7 & 7.7 & \multirow[t]{9}{*}{43.90} & \multirow[t]{9}{*}{$.000 * * *$} \\
\hline Substance use disorders & 40 & 10.4 & 2 & 2.2 & & \\
\hline Schizophrenia and related psychosis & 190 & 49.5 & 38 & 41.8 & & \\
\hline Mood disorders & 55 & 14.3 & 32 & 35.2 & & \\
\hline Mania and bipolar disorders & 24 & 6.2 & 12 & 13.2 & & \\
\hline Depression and related disorders & 31 & 8.1 & 20 & 22.0 & & \\
\hline Anxiety and stress-related disorders & 40 & 10.4 & 6 & 6.6 & & \\
\hline Personality disorders & 42 & 10.9 & I & I.I & & \\
\hline $\begin{array}{l}\text { Other disorders (i.e. eating disorders, } \\
\text { mental retardation, other mental } \\
\text { disorders) }\end{array}$ & 4 & 1.0 & 5 & 5.5 & & \\
\hline $\begin{array}{l}\text { Suicide attempt and/or self-harm } \\
\text { before admission }\end{array}$ & 61 & 15.9 & 17 & 18.7 & 0.41 & .524 \\
\hline \multirow[t]{3}{*}{ Overt aggression before admission } & 67 & 17.7 & 17 & 19.3 & 0.12 & .726 \\
\hline & \multirow{2}{*}{\multicolumn{2}{|c|}{$\begin{array}{l}\mathrm{BKR}(N=465) \\
M \pm \mathrm{SD}\end{array}$}} & \multirow{2}{*}{\multicolumn{2}{|c|}{$\begin{array}{l}\mathrm{HH}(N=91) \\
M \pm \mathrm{SD}\end{array}$}} & \multicolumn{2}{|l|}{ t-test } \\
\hline & & & & & $t$ & $p$ \\
\hline GAF at admission ${ }^{a}$ & \multicolumn{2}{|c|}{$43.5 \pm 17.4$} & \multicolumn{2}{|c|}{$29.3 \pm 13.2$} & 8.59 & $.000 * * *$ \\
\hline CGI Part I at admission ${ }^{b}$ & \multicolumn{2}{|c|}{$5.9 \pm 1.1$} & \multicolumn{2}{|c|}{$6.1 \pm 1.0$} & -1.96 & .051 \\
\hline
\end{tabular}

$* * * p<.001$.

a GAF is scored from 0 to 100 . A higher score indicates better psychosocial functioning.

bCGI Part I describes the severity of illness, with scores ranging from I to 8.A higher score indicates a more severe degree of illness.

\section{Discussion}

\section{Overview of mental health care services in Germany and Japan}

In Germany, there is universal coverage for mental health services, and they are provided by both private and public institutions (Gaebel, Janssen \& Zielasek, 2009). Approximately $10 \%$ of all health care spending goes to mental health care (OECD, 2008). The national government provides a basic legal framework by passing general health care or welfare legislation. However, planning and regulating mental health care is the responsibility of the 16 federal states. As a result, the provision of German health care, particularly mental health care, is spread among many sectors and characterized by considerable differences (Salize, Rössler \& Becker, 2007). Following a psychiatry reform in the mid-1970s, hospital-based services were transformed to community and outpatient services. Psychiatric hospitals changed their focus towards regionalized acute hospital care alongside a growing number of psychiatric wards at general hospitals (Salize et al., 2007). The number of admissions has increased, concurrent with shorter length of stay $(770,514$ cases, 38.4 days in 1994 vs 1,127,971 cases, 20.3 days in 2008) (FHR, 2008a). Of all admissions, substance use disorders are the most common diagnostic group, followed by affective disorders, anxiety disorders and schizophrenia in descending order (FHR, 2008b). The proportion of involuntary admissions has been rather stable over time representing $14-17 \%$ of all psychiatric admissions (Salize et al., 2007), despite an increase from 114.4 cases per 100,000 population in 1990 to 237.2 in 2006 (Priebe et al., 2008), representing a trend seen in parts of other European countries (Priebe et al., 2008; Salize \& Dressing, 2004).

In Japan, there is also universal coverage for mental health services. Although health care providers are predominantly private, macro-regulation by government shapes the Japanese health care system (Ito, 2009). A total of $6.8 \%$ of all health care spending goes to mental health care (OECD, 2008). Japanese mental health services, once known for having a large number of psychiatric beds and long length of stay (Shinfuku, 1998), have been criticized for the excessive number of inpatients, insufficient community resources and infringement on the human rights of the mentally ill (Mino, Kodera \& Bebbington,1990). In response, since the 1990s, there has been a major policy shift from hospitals to the community by shortening the length of stay, discharging long-stay patients and developing community services (Ito, 2009). In this context, outcome assessment including length of stay and readmission 
Table 3. Psychopharmacological and psychosocial treatment during inpatient stay.

\begin{tabular}{|c|c|c|c|c|c|c|}
\hline & \multicolumn{2}{|c|}{$\operatorname{BKR}(N=465)$} & \multicolumn{2}{|c|}{$\mathrm{HH}(\mathrm{N}=91)$} & \multicolumn{2}{|l|}{$\chi^{2}$ test } \\
\hline & $n$ & $\%$ & $n$ & $\%$ & $\chi^{2}$ & $p$ \\
\hline Psychopharmacological treatment & 297 & 80.5 & 78 & 100 & 18.14 & $.000 * * *$ \\
\hline Typical antipsychotics & 147 & 39.8 & 35 & 44.9 & 0.68 & .411 \\
\hline High potency & $11 \mathrm{I}$ & 30.1 & 8 & 10.3 & 12.95 & $.000 * * *$ \\
\hline Low potency & 80 & 21.7 & 30 & 38.5 & 9.77 & $.000 * * *$ \\
\hline Depot application (injection) & 43 & 11.7 & 2 & 2.6 & 5.88 & .015 \\
\hline Atypical antipsychotics & 161 & 43.6 & 62 & 79.5 & 33.11 & $.000 * * *$ \\
\hline Clozapine ${ }^{a}$ & 32 & 8.7 & 0 & 0 & 7.29 & .007 \\
\hline Olanzapine & 51 & 13.8 & 19 & 24.4 & 5.41 & .020 \\
\hline Risperidone & 41 & II.I & 32 & 41.0 & 42.17 & $.000 * * *$ \\
\hline $\begin{array}{l}\text { Others (quetiapine, aripiprazole, amisulpride, } \\
\text { b ziprasidone, }{ }^{\mathrm{b}} \text { sertindole, }{ }^{\mathrm{b}} \text { zotepine) }\end{array}$ & 63 & 17.1 & 41 & 52.6 & 45.43 & $.000 * * *$ \\
\hline \multicolumn{7}{|l|}{ Antidepressants } \\
\hline Tricyclics & 24 & 6.5 & 9 & 11.5 & 2.39 & .122 \\
\hline $\begin{array}{l}\text { SSRIs (citalopram, }{ }^{b} \text { escitalopram, },^{c} \text { fluoxetine, }{ }^{b} \\
\text { fluvoxamine, paroxetine, sertraline) }\end{array}$ & 38 & 10.3 & 22 & 28.2 & 17.77 & $.000 * * *$ \\
\hline Other antidepressants & 24 & 6.5 & 8 & 10.3 & 1.36 & .243 \\
\hline \multicolumn{7}{|l|}{ Mood stabilizers } \\
\hline Lithium salts & 14 & 3.8 & 6 & 7.7 & 2.29 & .130 \\
\hline Carbamazepine & 9 & 2.4 & 6 & 7.7 & 5.48 & .019 \\
\hline Valproic acid & 30 & 8.1 & 21 & 26.9 & 22.50 & $.000 * * *$ \\
\hline $\begin{array}{l}\text { Other mood stabilizers/other antiepileptics (lamotrigine, }{ }^{\mathrm{d}} \\
\text { topiramate, }{ }^{\mathrm{e}} \text { pregabalin }{ }^{\mathrm{e}} \text { ) }\end{array}$ & 44 & 12.2 & 0 & 0 & 10.58 & .001 \\
\hline Anxiolytics and/or hypnotics & 85 & 23.0 & 75 & 96.2 & 149.79 & $.000 * * *$ \\
\hline Anticholinergics & 9 & 2.4 & 32 & 41.0 & 115.08 & $.000 * * *$ \\
\hline \multicolumn{7}{|l|}{ Number of classes of psychotropics } \\
\hline One & 74 & 20.1 & I & $\mathrm{I} .3$ & 193.64 & $.000 * * *$ \\
\hline Two & 153 & 42.5 & 14 & 17.9 & & \\
\hline Three & 105 & 28.5 & 42 & 53.8 & & \\
\hline Four or more & 5 & 1.4 & 20 & 25.6 & & \\
\hline \multicolumn{7}{|l|}{ Number of antipsychotics } \\
\hline One & 136 & 36.9 & 28 & 35.9 & 16.05 & .001 \\
\hline Two & 84 & 22.8 & 26 & 33.3 & & \\
\hline Three or more & 37 & 10.0 & 15 & 19.2 & & \\
\hline \multicolumn{7}{|l|}{ Number of antidepressants } \\
\hline One & 64 & 17.3 & 24 & 30.0 & 14.80 & .001 \\
\hline Two or more & II & 3.0 & 7 & 9.0 & & \\
\hline Problems related to pharmacological therapy & 29 & 7.8 & I & 1.2 & & \\
\hline \multicolumn{7}{|l|}{ Psychotherapy } \\
\hline Supportive psychotherapy (i.e. no specific therapy) & 87 & 23.6 & 81 & 92.0 & 1.44 & $.000 * * *$ \\
\hline Cognitive behavioural therapy & 20 & 5.4 & 0 & 0 & 4.97 & .026 \\
\hline Special therapy for substance or alcohol dependence & 15 & 4.1 & I & 1.2 & 1.80 & .180 \\
\hline Psychoeducation & 13 & 3.5 & 4 & 4.5 & 0.21 & .645 \\
\hline Problems related to psychotherapy & 12 & 3.3 & 18 & 20.5 & 34.16 & $.000 * * *$ \\
\hline \multicolumn{7}{|l|}{ Other therapeutic measures } \\
\hline Occupational therapy & 182 & 49.6 & 29 & 34.6 & 6.05 & .014 \\
\hline Sport therapy & 21 & 5.7 & 24 & 27.9 & 38.33 & $.000 * * *$ \\
\hline Socio-therapeutic measures & 51 & 13.9 & 18 & 20.9 & 2.67 & .102 \\
\hline
\end{tabular}

$* * * p<.001$.

aNot available in Japan until 2009.

bNot available in Japan.

cNot available in Japan until 20I I.

dNot available in Japan until December 2008.

eNot available in Japan until 2010. 
Table 4. Outcomes and characteristics at discharges.

\begin{tabular}{|c|c|c|c|c|c|c|c|c|}
\hline & \multicolumn{3}{|c|}{ BKR $(N=465)$} & \multicolumn{3}{|c|}{$\mathrm{HH}(\mathrm{N}=9 \mathrm{I})$} & \multicolumn{2}{|l|}{ t-test } \\
\hline & M & SD & Median & M & SD & Median & $t$ & $p$ \\
\hline \multirow[t]{2}{*}{ Length of stay (days) } & 28.4 & 30.2 & 19 & 75.2 & 58.1 & 64 & -7.24 & $.000 * * * *$ \\
\hline & & $n$ & $\%$ & & $n$ & $\%$ & & \\
\hline $1-3$ & & 94 & 20.5 & & 0 & 0 & $\chi^{2}=87.93$ & $.000 * * * *$ \\
\hline $4-30$ & & 202 & 44.1 & & 19 & 22.4 & & \\
\hline $31-90$ & & 138 & 30.1 & & 38 & 44.9 & & \\
\hline More than 90 & & 24 & 5.2 & & 28 & 32.9 & & \\
\hline GAF at discharge ${ }^{a}$ & 59.9 & 16.0 & 60 & 58.1 & 12.7 & 60 & -1.12 & .263 \\
\hline$\triangle$ GAF (GAF at discharge - GAF at admission) & 15.8 & 19.2 & 15 & 27.7 & 18.0 & 30 & -5.03 & $.000 * *$ \\
\hline CGI Part $I^{\mathrm{b}}$ at discharge & 5.3 & 1.1 & 5 & 4.2 & 1.0 & 4 & 8.50 & $.000 * *$ \\
\hline \multirow[t]{3}{*}{ CGI Part IIc at discharge } & 3.5 & 0.8 & 3 & 2.9 & 0.9 & 3 & 5.26 & $.000^{* *}$ \\
\hline & \multicolumn{2}{|c|}{ BKR $(N=465)$} & & \multicolumn{3}{|c|}{$\mathrm{HH}(\mathrm{N}=91)$} & \multicolumn{2}{|l|}{$\chi^{2}$ test } \\
\hline & $n$ & $\%$ & & $n$ & & $\%$ & $\chi^{2}$ & $p$ \\
\hline Type of discharge & & & & & & & 2.04 & .564 \\
\hline Regular & 327 & 88.1 & & 79 & & 92.9 & & \\
\hline Temporary transfer & 11 & 3.0 & & 1 & & 1.2 & & \\
\hline Against medical advice & 22 & 5.9 & & 4 & & 4.7 & & \\
\hline Critical accident & 11 & 3.0 & & 1 & & 1.2 & & \\
\hline Suicide attempt and/or self-harm during stay & 8 & 2.2 & & 4 & & 4.6 & 1.65 & .199 \\
\hline Overt aggression during stay & 26 & 7.0 & & 2 & & 2.2 & 2.88 & .090 \\
\hline Restraint during stay & 31 & 8.4 & & 14 & & 15.7 & 4.35 & .037 \\
\hline Inpatient aftercare & & & & & & & 7.05 & .133 \\
\hline None & 343 & 93.0 & & 77 & & 91.7 & & \\
\hline Other department in the present hospital & 7 & 1.9 & & I & & 1.2 & & \\
\hline Other psychiatric hospital & 12 & 3.3 & & 3 & & 3.6 & & \\
\hline Other general hospital & 5 & 1.4 & & 0 & & 0 & & \\
\hline Others & 2 & 0.5 & & 3 & & 3.6 & & \\
\hline \multicolumn{9}{|l|}{ Outpatient aftercare } \\
\hline None & 61 & 16.4 & & 10 & & 12.3 & 0.84 & .359 \\
\hline GP & 100 & 27.0 & & I & & 1.2 & 25.35 & $.000 * * * *$ \\
\hline Psychiatrist in private practice & 181 & 48.8 & & 15 & & 18.5 & 24.80 & $.000 * * * *$ \\
\hline Psychotherapist & 18 & 4.9 & & 0 & & 0 & 4.09 & .043 \\
\hline Outpatient department of the present psychiatric hospital & 49 & 13.2 & & 28 & & 34.6 & 21.46 & $.000 * * * *$ \\
\hline Outpatient department of another psychiatric hospital & 5 & 1.3 & & 28 & & 34.6 & 108.41 & $.000 * * * *$ \\
\hline Psychosocial service & 7 & 1.9 & & 0 & & 0 & 1.55 & .213 \\
\hline Other practitioner & 5 & 1.3 & & 1 & & 1.2 & 0.01 & .934 \\
\hline Others (i.e. advice centre, self-help group) & 6 & 1.6 & & 1 & & 1.2 & 0.07 & .792 \\
\hline Housing situation after discharge & & & & & & & 22.39 & $.000^{* * * *}$ \\
\hline At home & 289 & 80.1 & & 73 & & 89.0 & & \\
\hline Care home or supported housing & 59 & 16.3 & & 2 & & 2.4 & & \\
\hline Homeless & 7 & 1.9 & & 0 & & 0 & & \\
\hline Others & 6 & 1.7 & & 7 & & 8.5 & & \\
\hline Diagnosis at discharge & & & & & & & 37.21 & $.000^{* * * *}$ \\
\hline Organic brain disorders (including dementia) & 16 & 3.6 & & 5 & & 6.3 & & \\
\hline Substance use disorders & 38 & 8.6 & & 3 & & 3.8 & & \\
\hline Schizophrenia and related psychosis & 218 & 49.4 & & 31 & & 38.8 & & \\
\hline Mood disorders & 66 & 15.0 & & 30 & & 37.5 & & \\
\hline Mania and bipolar disorders & 28 & 6.3 & & II & & 13.8 & & \\
\hline Unipolar depression and related disorders & 38 & 8.6 & & 19 & & 23.8 & & \\
\hline Anxiety and stress-related disorders & 47 & 10.7 & & 6 & & 7.5 & & \\
\hline Personality disorders & 51 & 11.6 & & 1 & & 1.3 & & \\
\hline $\begin{array}{l}\text { Other disorders (i.e. eating disorders, mental retardation, } \\
\text { other mental disorders) }\end{array}$ & 5 & 1.1 & & 4 & & 5.0 & & \\
\hline
\end{tabular}

$* * * p<.001$

aGAF is scored from 0 to 100.A higher score indicates better psychosocial functioning.

${ }^{\mathrm{b}} \mathrm{CGI}$ Part I describes the severity of illness, with scores ranging from I to 8.A higher score indicates a more severe degree of illness.

${ }^{c} \mathrm{CGI}$ Part II describes the improvement/deterioration during stay. A lower score indicates greater improvement of illness. 
Table 5. Treatment process and outcomes for schizophrenia over time.

\begin{tabular}{|c|c|c|c|c|}
\hline & \multicolumn{2}{|c|}{ German hospital } & \multicolumn{2}{|c|}{ Japanese hospital } \\
\hline & $\begin{array}{l}2008^{a} \\
\text { (\% or Mean) }\end{array}$ & $\begin{array}{l}1997^{\mathrm{b}} \\
\text { (\% or Mean) }\end{array}$ & $\begin{array}{l}2008^{c} \\
\text { (\% or Mean) }\end{array}$ & $\begin{array}{l}1997^{d} \\
\text { (\% or Mean) }\end{array}$ \\
\hline \multicolumn{5}{|l|}{ Psychopharmacological treatment } \\
\hline Neuroleptic drugs & 97 & 96 & 97 & 98 \\
\hline Atypical antipsychotics & 65 & 18 & 97 & 12 \\
\hline Antidepressants & 9 & 16 & 7 & 8 \\
\hline Benzodiazepines & 27 & 17 & 93 & 78 \\
\hline Other tranquillizer & 9 & I & 20 & 24 \\
\hline Lithium salts & 2 & 5 & 3 & 7 \\
\hline Carbamazepine & 2 & 4 & 3 & 18 \\
\hline Valproic acid & 7 & 2 & 17 & 16 \\
\hline Anticholinergics & 3 & 13 & 73 & 96 \\
\hline \multicolumn{5}{|l|}{ Psychosocial treatment } \\
\hline Occupational therapy & 59 & 76 & 33 & 52 \\
\hline Sport therapy & 7 & 42 & 3 & 0 \\
\hline Socio-therapeutic measures & 17 & 18 & 17 & 2 \\
\hline Length of stay (days) & 36 & 52 & 81 & 150 \\
\hline$\triangle$ GAF (GAFe at discharge - GAF at admission) & 15.2 & 16.6 & 31.1 & 22.2 \\
\hline CGI Part IIf at discharge & 3.4 & 3.5 & 2.8 & 3.2 \\
\hline
\end{tabular}

${ }^{a}$ Admissions to the BKR diagnosed at discharge as schizophrenia and related psychosis in this study $(n=218)$.

bPatients with schizophrenia admitted to the BKR in $1997(n=856)$ (Hübner-Liebermann, SpießI, Iwai et al., 2005).

cAdmissions to the $\mathrm{HH}$ diagnosed at discharge as schizophrenia and related psychosis in this study $(n=3 \mathrm{I})$.

dPatients with schizophrenia admitted to the Tokyo Women's Medical University in $1997(n=50)$ (Hübner-Liebermann, Spießl, Iwai et al., 2005).

eGAF is scored from 0 to 100 . A higher score indicates better psychosocial functioning.

${ }_{\mathrm{f}} \mathrm{CGI}$ Part II describes the improvement/deterioration during stay. A lower score indicates greater improvement of illness.

rate have been introduced in acute psychiatric inpatient care units/wards, in which reimbursement is double or triple that for the standard inpatient unit (Ito, 2009). The average length of stay for discharged patients from Japanese psychiatric hospitals is still very long (396.3 days in 2008) (JMHLW, 2008a). However, the average length of stay in acute psychiatric wards is considerably shorter, generally less than 90 days. Schizophrenia and related psychosis accounts for approximately $70 \%$ of all inpatients in psychiatric hospitals, followed by organic brain disorders (mainly dementia) and affective disorders (JMHLW, 2008a). In Japan, the total number of involuntary admissions has increased from 71.0 cases per 100,000 population in 1993 to 155.7 in 2008 (JMHLW, 2008b). This has been primarily a result of the increased number of admissions under guardianship, where a partner or relative of the patient usually becomes the guardian. Involuntary admission by guardianship is now an issue of discussion in Japan.

\section{Acute admission settings}

During the index period, 465 and 91 acute admissions were documented for the BKR and the $\mathrm{HH}$, respectively. Although both hospitals provide nearly the same number of beds for acute admissions, the BKR received five times more admissions, including more re-hospitalization cases with shorter length of stay than the HH. This difference could be attributed to the dissimilarity of the regional emergency systems and the length of stay. Admissions to the BKR were more frequently voluntary than those to the HH. Threatening behaviour or actual danger to oneself or to others is a common criterion for involuntary admission in both countries. In addition, the need for treatment but lack of insight is a criterion for involuntary admissions by guardianship in Japan. This might have contributed to the higher prevalence of involuntary admissions to the $\mathrm{HH}$.

Consistent with another study (Preti et al., 2009), the current study suggests that schizophrenia and related psychosis is the most common diagnosis in acute admission settings regardless of differences in region and type of facilities. However, the BKR had a wider diagnostic spectrum, as shown by the higher proportion of admissions with substance use disorders, anxiety disorders and personality disorders. Patients with substance use disorders were extremely rare in the HH. In Japan, patients with substance use disorders are treated in special wards/departments after acute admission settings, as they are in Germany. However, these disorders account for only $4.5 \%$ of all inpatients in Japanese psychiatric hospitals (JMHLW, 2008a), in contrast to $37.8 \%$ of all admissions to German psychiatric hospitals (FHR, 2008). The difference in proportions of patients hospitalized with substance use disorders may be 
attributed to the lower number of patients who present with such disorders in Japan compared to Germany.

Admissions to the BKR were characterized by a shorter period since onset of the present episode, cases referred from the police or the health authorities and cases without prior treatment before admission. These findings suggest that admissions to the BKR are more often arranged as crisis interventions and as the primary entry or re-entry point into the network of psychiatric services. Unplanned or acute admissions typically have a shorter length of stay than planned admissions because not many days in the hospital are needed when the treatment goal is to resolve the crisis (Øiesvold et al., 1999). In fact, a fifth of all admissions to the BKR were discharged within three days, and the majority of those admitted received a non-psychotic diagnosis (substance use disorders: 25.3\%; anxiety disorders: $32.2 \%$; personality disorders: $16.1 \%$ ). In contrast, most patients in the $\mathrm{HH}$ had already received outpatient treatment during the relatively longer process leading to admission, with their admission likely initiated by doctors and psychosocial services of hospitals. These findings suggest that admissions to the $\mathrm{HH}$ are more often arranged for patients who did not improve with prior treatment, patients with more impairment of psychosocial functioning (representing lower GAF at admission) and patients with schizophrenia and mood disorders, accounting for about $80 \%$ of all admissions to the $\mathrm{HH}$.

\section{Process and outcomes of acute inpatient care}

Psychopharmacological treatment is considered a main part of acute inpatient care. The high proportion of antipsychotics used in both hospitals can be attributed to the high proportion of admissions with schizophrenia and related psychosis. Moreover, antipsychotics might be applied to cases with acute psychotic symptoms, agitation or who represent a danger to themselves or others, which are common reasons for the acute admission setting. Like other previous studies (Grohmann, Engel, Geissler \& Ruther, 2004; Tan, Shinfuku \& Sim, 2008), our study suggests a trend of increasing use of atypical antipsychotics and newer antidepressants such as SSRIs. A large number of psychotropics does not necessarily imply polypharmacy; the database lists the medications used during the stay, but not the sequence or duration. Although more psychotropics as a whole were administered in the $\mathrm{HH}$, problems related to psychopharmacological treatment were rare.

The limited implementation of psychosocial interventions, especially in the BKR, might have resulted from the predominant strategies of psychopharmacological treatment, since it is considered to be more beneficial over a short time span. However, patients admitted to acute departments often exhibit not only medical problems, but also psychosocial problems (Larkin et al., 2009). In general, psychotherapy in both hospitals seemed to lack variety.
Problems related to psychotherapy such as insufficient compliance were observed more frequently at the $\mathrm{HH}$, where supportive psychotherapy was applied to almost all cases. Application of other psychotherapies was rare in this study. However, CBT is proposed not only for patients in the community, but also for acute inpatient care (McCann $\&$ Bowers, 2005). In addition, brief interventions developed in emergency departments for patients with substance use disorders provide a model for the development of similar interventions for people with mental health problems (Larkin et al., 2009). Moreover, psychosocial interventions such as psychoeducation can prevent relapse and re-hospitalization.

Differences in acute admission settings and treatment strategies between the two hospitals are consequently assumed to have influenced outcomes. A review pointed out that length of stay is influenced by the process of treatment and organizational characteristics of institutions, rather than by socio-demographic and diagnostic factors (Richter, 2001). In this study, the $\mathrm{HH}$, providing more extensive psychopharmacological and psychosocial treatment, required prolonged hospitalizations, while the BKR with more emergency admissions had shorter hospitalizations. As a result, the $\mathrm{HH}$ with its longer duration of hospitalization could achieve greater improvements in GAF and CGI.

\section{Changes in treatment for schizophrenia over 10 years}

The $49.4 \%$ of admissions to the BKR $(n=218)$ and $38.3 \%$ to the $\mathrm{HH}(n=31)$ diagnosed at discharge as schizophrenia and related psychosis (Table 4) were compared to our previous study based on a sample collected in 1997 (HübnerLiebermann, Spieß1, Iwai et al., 2005) (Table 5). This study had some methodological differences from the previous one, in that the sample of the present study was collected in a different Japanese hospital and only in acute wards of both hospitals. However, we were able to evaluate broad changes in treatment for schizophrenia in a German and a Japanese hospital over 10 years. Prescriptions for atypical antipsychotics increased dramatically in both hospitals and might reflect decreased prescriptions for anticholinergics. The remarkably higher proportion of benzodiazepines and anticholinergics administered in the Japanese hospital is consistent with the previous study. In addition, some studies of patients with schizophrenia (Bitter et al., 2003; Kuroda et al., 2008) have suggested that the use of these medications in Japan is high, from an international point of view. More frequent use of anticholinergics in the $\mathrm{HH}$ is assumed to be related to higher sensitivity for extrapyramidal symptoms among Asian patients (Ishigooka, Inada \& Miura, 2001). The previous study revealed that non-pharmacological treatment was implemented less in the Japanese hospital. This study 
involved only acute admissions, with psychosocial treatment generally less used in both hospitals. However, socio-therapeutic measures have become more available in Japan over the 10-year period. The average length of stay decreased in both hospitals. Consistent with the previous study, greater improvement in GAF and CGI was achieved with longer duration of hospitalization in the Japanese hospital.

\section{Outpatient aftercare}

Outpatient care after discharge from the BKR was usually provided through a general practitioner (GP) or a psychiatrist in private practice. The GP plays an important role as a gatekeeper in the German mental health care system. A previous study (Hübner-Liebermann, Spießl, \& Cording, 2005) suggested that patients with substance use disorders and elderly patients tend to receive aftercare from a GP compared to patients with schizophrenia or mood disorders who receive it from a psychiatrist in private practice. As a rule, outpatient departments of psychiatric hospitals in Germany provide specific treatment only for patients with severe and persistent mental disorders who need multi-professional community care (Salize et al., 2007). However, the problematic interface between outpatient and inpatient care, which are funded separately and staffed by different teams, has been pointed out (Salize et al., 2007), and a need for constructive collaboration has been recognized (Spieß1 \& Cording, 2007). In contrast, in the HH, outpatient aftercare is mainly provided by the outpatient department of psychiatric hospitals. In the Japanese health care system, there is no assigned catchment area even in primary care (Ito, 2009), and GPs differ from German practitioners in that they rarely treat patients with mental illness. Although the number of private psychiatric practices has increased rapidly in Japan (Shinfuku, 1998), patients who need inpatient treatment tend to be treated after discharge in the outpatient department of psychiatric hospitals. This tendency suggests that Japanese psychiatric service provision is still based in hospitals because of insufficient community-based services. However, aftercare provided by the patient's same therapist might be considered an advantage.

\section{Limitations}

This study attempted to assess and compare acute inpatient services using data of the BADO, designed as a screening tool to determine the quality of psychiatric inpatient care in Germany. For international comparability, further validation is needed regarding whether the Japanese version (J-BADO) is adequate for the Japanese mental health system. Our two hospitals in this study could not represent the characteristics of all hospitals in each country. Based on data from the two hospitals, conclusions regarding health care systems should be understood as representing a proposal that requires replication in other evaluations.

\section{Conclusions}

For acute admissions in Germany, intensive care with short duration of hospitalization is provided as crisis intervention. In contrast, comprehensive care preceding emergency admission settings is provided in Japan, and greater improvement is achieved with longer hospitalization. It is impossible to assess definitively which of the two systems represents a well-functioning psychiatric service. However, provision of an acute admission setting without delay when needed can prevent aggravation and more impairment of psychosocial functioning. Moreover, improvement of the treatment process may avoid re-hospitalization. It is important not to promote deinstitutionalization by only reducing psychiatric beds and shortening the length of stay; establishing a sufficient community service system is also important (Munk-Jørgensen, 1999). To achieve this, it is necessary to evaluate the quality and role of acute inpatient care in different service contexts. Our results may contribute to the improvement of the quality of psychiatric services.

\section{References}

Appleby, L., Desai, P. N., Luchins, D. J., Gibbons, R. D., \& Hedeker, D. R. (1993). Length of stay and recidivism in schizophrenia: A study of public psychiatric hospital patients. American Journal of Psychiatry, 150, 72-76.

Bitter, I., Chou, J. C., Ungvari, G. S., Tang, W. K., Xiang, Z., Iwanami, A., \& Gaszner, P. (2003). Prescribing for inpatients with schizophrenia: An international multi-center comparative study. Pharmacopsychiatry, 36, 143-149.

Cording, C. (1998). Conceptual aspects in development and implementation of basic psychiatric documentation. Psychiatrische Praxis, 25, 175-178 [in German].

FHR (Federal Health Reporting). (2008a). Diagnostic data of the hospitals for Germany starting from 1994 (cases/deaths, days of care, average length of stay). Berlin: FHR.

FHR (Federal Health Reporting). (2008b). Diagnostic data of the hospitals starting from 2000 (cases/deaths, days of care, average length of stay). Berlin: FHR.

Gaebel, W., Janssen, B., \& Zielasek, J. (2009). Mental health quality, outcome measurement, and improvement in Germany. Current Opinion in Psychiatry, 22, 636-642.

Goldstein, G. \& Shemansky, W. J. (2000). Length and number of hospitalizations in two cohorts of veterans with chronic schizophrenia. Psychiatric Services, 51, 245-247.

Grohmann, R., Engel, R. R., Geissler, K. H., \& Ruther, E. (2004). Psychotropic drug use in psychiatric inpatients: Recent trends and changes over time - data from the AMSP study. Pharmacopsychiatry, 37 Suppl 1, 27-38.

Hübner-Liebermann, B., Spieß1, H., \& Cording, C. (2005). Who comes from where and who goes where? Treatment methods for psychiatric inpatients. Nervenarzt, 76, 856-864 [in German].

Hübner-Liebermann, B., Spieß1, H., Iwai, K., \& Cording, C. (2005). Treatment of schizophrenia: Implications derived from an intercultural hospital comparison between Germany and Japan. International Journal of Social Psychiatry, 51, 83-96. 
Ishigooka, J., Inada, T., \& Miura, S. (2001). Olanzapine versus haloperidol in the treatment of patients with chronic schizophrenia: Results of the Japan multicenter, double-blind olanzapine trial. Psychiatry and Clinical Neurosciences, 55, 403-414.

Ito, H. (2009). Quality and performance improvement for mental healthcare in Japan. Current Opinion in Psychiatry, 22, 619-622.

JMHLW (Japan Ministry of Health, Labour and Welfare). (2008a). Patient survey 2008. Tokyo: Statistics and Information Department, MHW [in Japanese].

JMHLW (Japan Ministry of Health, Labour and Welfare). (2008b). Report on public health administration and services 2008. Tokyo: Kousei Toukei Kyokai [in Japanese].

Kuroda, N., Sun, S., Lin, C. K., Morita, N., Kashiwase, H., Yang, F., \& Nakatani, Y. (2008). Attitudes toward taking medication among outpatients with schizophrenia: Cross-national comparison between Tokyo and Beijing. Environmental Health and Preventive Medicine, 13, 288-295.

Larkin, G. L., Beautrais, A. L., Spirito, A., Kirrane, B. M., Lippmann, M. J., \& Milzman, D. P. (2009). Mental health and emergency medicine: A research agenda. Academic Emergency Medicine, 16, 1110-1119.

McCann, E., \& Bowers, L. (2005). Training in cognitive behavioural interventions on acute psychiatric inpatient wards. Journal of Psychiatric and Mental Health Nursing, 12, 215-222.

Mino, Y., Kodera, R., \& Bebbington, P. (1990). A comparative study of psychiatric services in Japan and England. British Journal of Psychiatry, 157, 416-420.

Moriwaki, K., Iwai, K., \& Cording, C. (2011). Quality management of inpatient care with basic documentation in German psychiatric hospitals. Seishin-Igaku, 53, 679-687 [in Japanese].

Munk-Jørgensen, P. (1999). Has deinstitutionalization gone too far? European Archives of Psychiatry and Clinical Neuroscience, 249, 136-143.

OECD (Organisation for Economic Co-operation and Development). (2008). Mental health in OECD countries. Policy Brief, November. Available from http://www.oecd.org/ dataoecd/6/48/41686440.pdf
Øiesvold, T., Saarento, O., Sytema, S., Christiansen, L., Göstas, G., Lönnerberg, O., Hansson, L. (1999). The Nordic Comparative Study on Sectorized Psychiatry: Length of in-patient stay. Acta Psychiatrica Scandinavica, 100, 220-228.

Oshima, I., Mino, Y., \& Inomata, Y. (2003). Institutionalisation and schizophrenia in Japan: Social environments and negative symptoms: Nationwide survey of in-patients. British Journal of Psychiatry, 183, 50-56.

Preti, A., Rucci, P., Santone, G., Picardi, A., Miglio, R., Bracco, R., de Girolamo, G. (2009). Patterns of admission to acute psychiatric in-patient facilities: A national survey in Italy. Psychological Medicine, 39, 485-496.

Priebe, S., Frottier, P., Gaddini, A., Kilian, R., Lauber, C., Martínez-Leal, R., Wright, D. (2008). Mental health care institutions in nine European countries, 2002 to 2006. Psychiatric Services, 59, 570-573.

Richter, D. (2001). Psychiatric inpatient length of stay: An overview of methods, influences and consequences. Fortschritte der Neurologie-Psychiatrie, 69, 19-31 [in German].

Salize, H. J., \& Dressing, H. (2004). Epidemiology of involuntary placement of mentally ill people across the European Union. British Journal of Psychiatry, 184, 163-168.

Salize, H. J., Rössler, W., \& Becker, T. (2007). Mental health care in Germany: Current state and trends. European Archives of Psychiatry and Clinical Neuroscience, 257, 92-103.

Shinfuku, N. (1998). Mental health services in Asia: International perspective and challenge for the coming years. Psychiatry and Clinical Neurosciences, 52, 269-274.

Spieß1, H., \& Cording, C. (2007). Collaboration of the general practitioner and the psychiatrist with the psychiatric hospital: A literature review. Fortschritte der Neurologie-Psychiatrie, 68, 206-215 [in German].

Spieß1, H., Hübner-Liebermann, B., Cording, C., \& Klein, H. E. (2004). Evidence-based hospital management considering data from the psychiatric basic documentation system. Psychiatrische Praxis, 31 Suppl 1, 9-11 [in German].

Tan, C. H., Shinfuku, N., \& Sim, K. (2008). Psychotropic prescription practices in east Asia: Looking back and peering ahead. Current Opinion in Psychiatry, 21, 645-650. 